International Journal of Wireless \& Mobile Networks (IJWMN) Vol. 5, No. 2, April 2013

\title{
DISCRETE COSINETRANSFORM-II FOR Reduction in Peak to Average Power Ratio OF OFDM SIGNALS THROUGH $\mu$-LAW COMPANDING TECHNIQUE
}

\author{
NavneetKaur and Lavish Kansal \\ Department of Electronics and Communication Engineering \\ Lovely Professional University, Phagwara \\ Punjab, India \\ nv_neet@yahoo.com \\ Department of Electronics and Communication Engineering \\ Lovely Professional University, Phagwara \\ Punjab, India \\ lavish.15911@lpu.co.in
}

\begin{abstract}
Orthogonal frequency Division multiplexing (OFDM) is the most familiar word in telecommunication and wireless communication systems as it provides enhanced spectral efficiency than Frequency division multiplexing (FDM).Although it is sustaining an orthogonal relation betweencarriers but high peak to average power ratio (PAPR) is one of the main disadvantages of OFDM system.Various PAPR reduction techniques have been used, including techniques based on companding. Incompanding, $\mu$-Law companding has potential to reduce the PAPR of OFDMsignals. $\mu$-Law Companding technique preserves the dynamic range of samples at low amplitudes.A new method named as precoding which is having less complexity compared to the other power reduction techniques is proposed to reduce PAPR. This paper put forward combination of two existing techniques namely $\mu$-Law Companding Transform and Discrete Cosine Transform-II precoding technique. The simulation results show that, the proposed combinedscheme gives better result for PAPR Reduction and results in no distortion.
\end{abstract}

Keywords

FDM, FDM, PAPR, FFT, IEEE, DCT, CCDF

\section{INTRODUCTION}

As the demand for high data rate wireless digital communication is rapidly expanding, several approaches such as increasing modulation order or employing multiple antennas at both transmitter and receiver that are reliable for wireless systems have been studied to enhance the spectral efficiency [1,2]. In today's communication systems Orthogonal Frequency Division Multiplexing (OFDM), widespread modulation technique has been considered to achieve high rate data transmission for wireless environment.OFDM system has high spectral efficiency, ease of implementation using the fast Fourier transform (FFT) and simple equalization techniques and also provides immunity to multipath fading, robustness against inter-symbol

DOI : 10.5121/ijwmn.2013.5211 
interference. Therefore it is considered for digital communication systems and also for future broadband applications.

Multicarrier networks such as Frequency Division Multiplexing (FDM) have been around since the late 1950's [3], however due to their implementation complexity transmitted in parallel. Multicarrier systems in wireless applications are less susceptible to channel induced distortions than single carrier systems at corresponding data rates and inefficient use of the frequency band they were restricted to military applications.

In multicarrier transmission, a single data stream bearing a large number of narrow-band subcarriers is transmitted over a number of lower rate subcarriers. Thesesubcarriers are regularly spaced in frequency, domain to avoid the interference. The subcarriers are totally independent of each other because thefrequency spacing and time synchronization of the subcarriers is chosen in such a waythat the subcarriers are orthogonal. By selecting a special set of orthogonal carrier frequencies, high spectral efficiency isobtained because the spectra of the sub-carriers do not overlap. The orthogonality among subcarriers can be maintained by introducing a cyclic prefix over a dispersive channel. The advantage of this structure over single carrier systems is that the extended symbol time due to lower data rate makes the signal less susceptible to effects of the channel such as multipath propagation which introduces Inter Symbol Interference (ISI). Each sub-channel will therefore experience flat fading reducing the equalization complexity in the receiver.

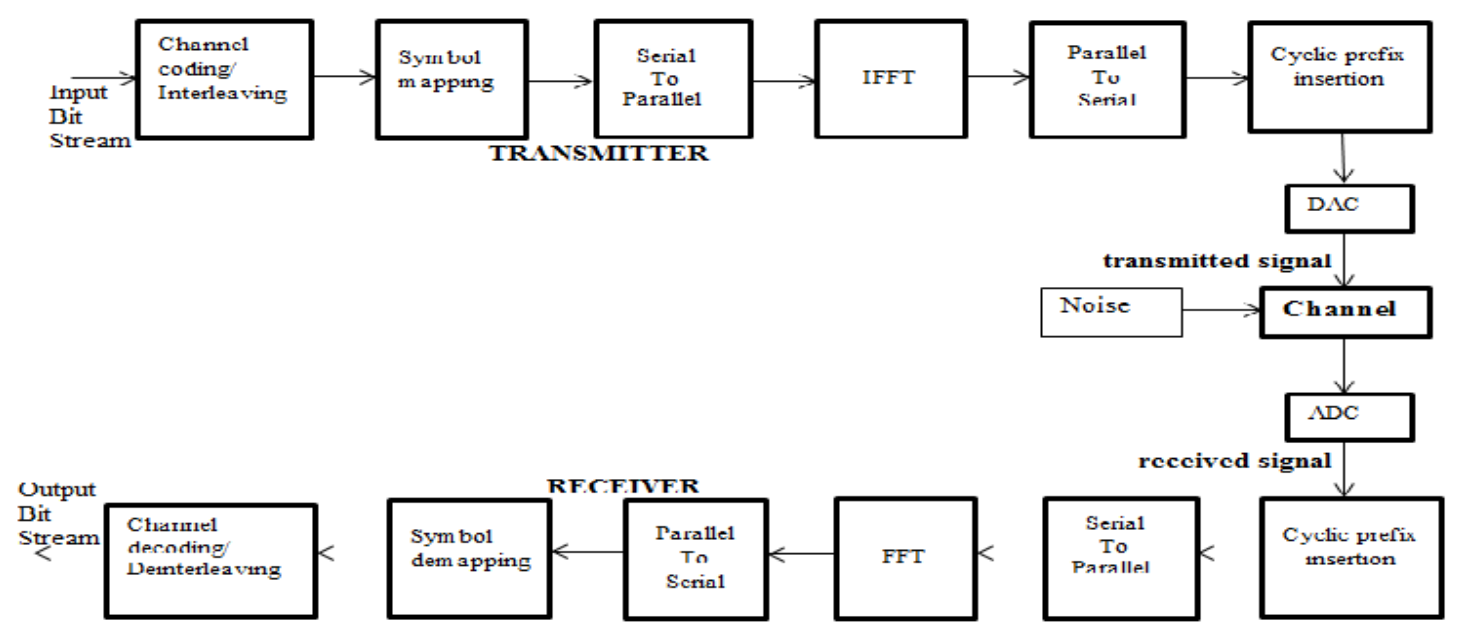

Fig. 1: Block Diagram of OFDM system

In an OFDM scheme shown in fig. 1, the orthogonal and overlapped subcarriers are a large number of orthogonal, overlapping, narrow band sub-carriers are transmitted in parallel.Maintaining total data rates similar to conventional single-carrier modulation schemes in the same bandwidth to modulate each sub-carrier is with a conventional modulation scheme such as Quadrature Amplitude Modulation (QAM) or Phase-Shift Keying (PSK) at a low symbol rate than that required for the whole data stream, but still.In OFDM system a high speed data is splits into a number of parallel lower data rate streams and these low rates data streams are transmitted simultaneously over a number of orthogonal subcarriers. The amount of dispersion in time caused due to multipath delay is reduced as, the symbol duration increases for lower rate parallel sub-carriers. These carriers divide the available transmission bandwidth. 
International Journal of Wireless \& Mobile Networks (IJWMN) Vol. 5, No. 2, April 2013

The sub-carriers are separated in such a way so that there is a very compact spectral utilization and each being modulated at a low bit rate. In a conventional frequency division multiplex the carriers are spaced apart to avoid spectral overlap and multipath distortion.

OFDM has progressed to the point where it has now been widely used for various communication applications such as digital audio and video broadcasting, asymmetric digital subscriber line modems, and wireless local area networks systems(WLAN).It has also been adopted as the physical layer modulation scheme for wireless networking standards such as Hiperlan2 in Europe and the Institute of Electrical and Electronic Engineers (IEEE) 802.11a, g standards in the United States.

OFDM systems have a large dynamic signal range with a very high peak to average power ratio (PAPR) which is a one of major drawbacks of the transmitted OFDM signal. As a result, the OFDM signal will be clipped when passed through a nonlinear high power amplifier (HPA) at the transmitter as it only operate in its linear region with sufficient back-off. Thus, HPA with a large dynamic range is required for OFDM systems. These amplifiers are very expensive and are major cost component of the OFDM systems. Thus reduction in the value of the PAPR not only reduces the cost of OFDM systems and reduces the complexity of analog-to-digital (A/D) and digital-to-analog (D/A) converters, but also increases the transmit power, thus, improving received signal-noise-ratio (SNR).

To reduce the PAPR, several techniques have been proposed, which can be divided into three groups.The first group of reduction techniques includes signal distortion techniques, which reduce the peak amplitudes simply by nonlinearly distorting the OFDM signal at or around the peaks. Examples of distortion techniques are clipping [5], peak windowing [6], and peak cancellation. Second, there are coding techniques that use a special code set that excludes OFDM symbols with a large PAPR. The special codes includes Golay complementary sequence [7], Reed Muller code [8], and Hadamard code in this approach [9]. The third technique scrambles each OFDM symbol with different scrambling sequences and selecting the sequence that gives the smallest PAPR such as selected mapping (SLM) [10] and partial transmit sequence (PTS) [11].

The organization of this paper is as follow. Section 2 presents formulates the problem of PAPR in OFDM system. The reduction technique based on $\mu$-Law companding is explained in section 3.Section 4 describes the precoding based Discrete Cosine Transform-II technique. PAPR schemes based on combined DCT-II and $\mu$-Law companding technique are proposed in section 5. Computer simulations results are presented in Section 6. Section 6 draws conclusions regarding the existing and proposed combined technique.

\section{PAPR IN OFDM SIGNAL}

Presence of large number of independently modulated sub-carriers in an OFDM system increasesthe peak value of the system as compared to the average of the whole system. The ratio of the peak to average power value is termed as Peak to Average Power Ratio.At some instant in time coherent addition of independently modulated $N$ sub-carriers same phase produces a peak:

$$
\operatorname{PAPR}[x(t)]=\frac{P_{P E A K}}{P_{A V E R A G E}}
$$


International Journal of Wireless \& Mobile Networks (IJWMN) Vol. 5, No. 2, April 2013

$$
=10 \log _{10} \frac{\max \left[|X(n)|^{2}\right]}{E\left[\left|x_{n}\right|^{2}\right]}
$$

Where $P_{\text {PEAK }}$ represents peak output power, $P_{A V E R A G E}$ means average output power. $\mathrm{E}[\cdot]$ denotesthe expected value, $x_{n}$ represents the transmitted OFDM signals which are obtained by takingIFFT operation on modulated input symbols is expressed as:

$$
x_{n}=\frac{1}{\sqrt{ } N} \sum_{i=0}^{N-1} X_{I} e^{j \frac{2 \pi}{N} n i}
$$

Where $N$ is the total number of subcarriers and the OFDM symbol duration is T seconds.

An OFDM symbol consists of $N$ subcarriers by the frequency spacing of $\Delta \mathrm{f}$. The total bandwidth $B$ of a symbol will be divided into $N$ equally spaced subcarriers and thus, all the subcarriers are orthogonal to each other within a time interval of length $T=1 / \Delta f$. A complex modulation symbol $X_{m, n}$, isused to modulate each sub-carrier independently where $m$ is a time index and $n$ is a subcarrier index.Then within the time interval $T$ the following signal of the $m$ th OFDM block period can be described as:

$$
x_{m}(t)=\frac{1}{\sqrt{N}} \sum_{N=0}^{N-1} X_{m, n} b_{n}(t-m T)
$$

Where, $b_{n}(\mathrm{t})$ is defined as:

$$
b_{n}(\mathrm{t})=\left\{\begin{array}{cc}
\exp (j 2 \pi n \Delta f t), & 0 \leq t \leq T \\
0, & \text { else }
\end{array}\right.
$$

Where, $b_{n}(\mathrm{t})$ is a rectangular pulse applied to each subcarrier. The total continuous time signal $x(t)$ consisting of all the OFDM block is given as:

$$
x(t)=\frac{1}{\sqrt{ } N} \sum_{m=0}^{\infty} \sum_{n=0}^{N-1} X_{m, n} g_{n(t-m T)}
$$

When compared with traditional single carrier systems, the instantaneous output of an OFDM system often has large fluctuations. To handle these large fluctuations the system devices, such as power amplifiers, A/Dconverters and D/A converters, must have large linear dynamic ranges. If the peak signal goes into the non-linearregion of devices at the transmitter,a series of undesirable interference is encountered, such as high out of band radiation and intermodulationdistortion. Therefore PAPR reduction techniques are requiredfor OFDM systems. The power output has large fluctuations due to which, the high power amplifier (HPA) should have largedynamic range. This results in poor power efficiency.

OFDM systems combine a number of independent sub carriers which causes increase in PAPR. The increase in PAPR is related to the number of sub carriers and their order of modulation. A curve called as Complementary Cumulative Distribution Function (CCDF)is used to present the vital information regarding the OFDM signal to be transmitted. These curves also provide the PAPR data needed by component designer. The main use of power CCDF curves is to identify the power characteristic of the signals which are amplified, mixed and decoded [12]. The plot of relative power levels of signal against their probability of occurrence is called CCDF curve. 
International Journal of Wireless \& Mobile Networks (IJWMN) Vol. 5, No. 2, April 2013

This curve illustrates the amount of time the signal remains at or above a given threshold level $[13,14]$. The ratio between power level and the average power is expressed in dBas:

$$
\mathrm{CCDF}=\operatorname{Prob}\left(\mathrm{PAPR}>P A P R_{o}\right)
$$

where $P A P R_{o}$ is the threshold level.

\section{3. $\mu$-LAW COMPanding TeChNiQUe}

In an OFDM system,large signals occur very infrequently therefore itis similar to thespeech signal, so the same companding technique can be used to improve the OFDM transmission performance. One of the companding technique named as $\mu$-Law Companding can effectively reduce the Peak to Average Power Ratio (PAPR) of the transmitted or the companded OFDM signals by transforming the statistics of the amplitudes of these signals into uniform distribution. The key idea of the $\mu$-law companderis that it covers all the encountered amplitudes of the OFDM signal. In $\mu$-law companding, the uniform distribution of the signals can be obtained by compressing the peak signals and expanding the small signals. In the process of companding, the amplitudes of the small signalsget enlarged, while the peaks remain unchanged. Therefore, the average power is increased and thus the PeaktoAverage Power Ratio (PAPR) can be reduced. This technique overcomes the deficiencies that are caused by clipping the original OFDM signal. The other advantage of the companding transform is that, it eliminates the Out of Band Interference (OBI) and can also maintain a constant average power level and also.

The original OFDM signal is converted into the companded signal by using the $\mathrm{Mu}$ Companding Transform. The companded signal obtained by using the MuCompanding Transform is given by the equation as:

$$
H(x)=\frac{\operatorname{sgn}(x) \ln (1+\mu|x|)}{\ln (1+\mu)}
$$

Where, $H(x)=$ Companded Signal obtained by $\mu$-LawCompandingTechnique, $\operatorname{sgn}(x)=\operatorname{sign}$ function, $x=$ original OFDM signal,

$\mu=255$ and it is the Mu-Law parameter of the compander, which controls the amount ofcompression.

\section{Discrete Cosine Transform-II}

The discrete cosine transforms (DCT) is a Fourier-related transform similar to the discrete Fourier transform (DFT). It is a member of a family of sinusoidal unitary transforms. This transform is real, orthogonal and separable with fast algorithms for its computation. They have abetter job of concentrating energy and compression of audio and data. The Discrete Cosine Transform (DCT) was first proposed by Ahmed et al. (1974) [15]. It is a Fourier-like transform, which equivalent to a DFT of roughly twice the length, operating on real data with even symmetry. The discrete cosine transform-II is a linear, invertible function $F: R_{n} \rightarrow R_{n}$; where $R$ denotes the set of real numbers, or equivalently an $n \times n$ square matrix. The $n$ real numbers $x_{0, . .}, x_{n-1}$ are transformed into the $n$ real numbers $f_{0, \ldots}, f_{n-1}$ according to one of the formulas: 


$$
f_{j}=\sum_{k=0}^{n-1} x_{k} \cos \left[\frac{\pi}{n} j\left(k+\frac{1}{2}\right)\right]
$$

The DCT-II implies the boundary conditions: $x_{k}$ is even around $k=-1 / 2$ and even around $k=n$ $1 / 2 ; f_{j}$ is even around $j=0$ and odd around $j=n$.

The DCT II is important because it has better computational efficiency, superiority in bandwidth compression of a wide range of signals, its powerful performance in the bit-rate reduction and robust against inter carrier interference (ICI). The precoding based techniques are simple and linear techniques with less complexity to implement without the need of any side information.

\section{Proposed ScheMe}

A new technique named as precoding technique is also used in OFDM systems to reduce the PAPR. Precoding techniques obtain a signal with lower PAPR than in the case of a without precoded OFDM system and it also reduces the interference produced by multiple users. The $\mu$-LawCompanding Transform also causes less spectrum side-lobes as compared to other reduction techniques.In proposed scheme $\mu$-Law companding was combined with precoding technique called Discrete Cosine Transform-II. The combined techniques based OFDM system was shown in fig. 2. In the transmitter, firstly DCT-II is used to transform the parallel data stream, and then the transformed data is processed by the companding unit. If data block passed by DCT-II, before IFFT, the PAPR is reduced, then the PAPR of OFDM signal could be further reduced by companding.

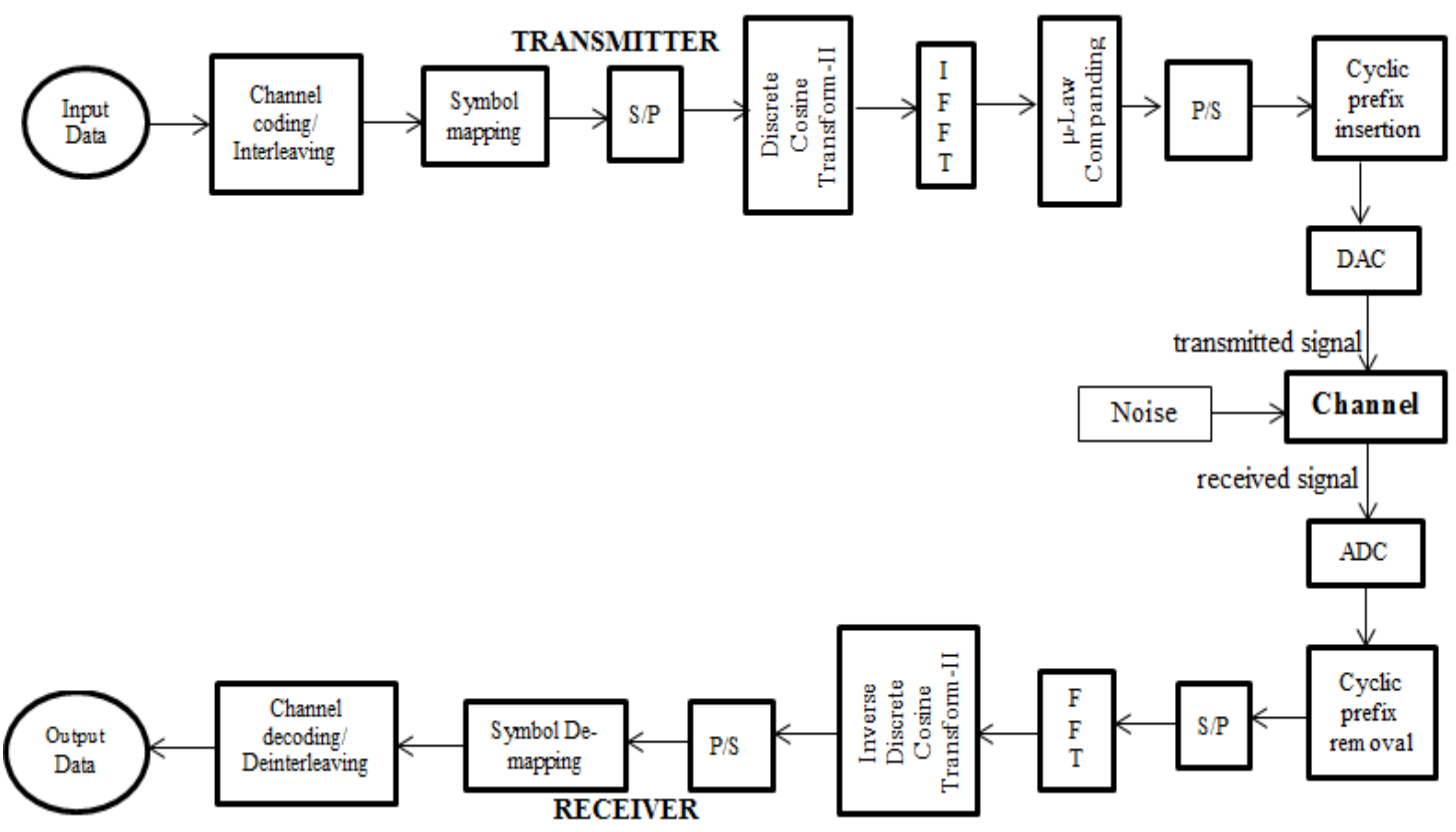

Fig. 2 Block scheme of DCT-II Precoding technique with $\mu$-law companding in OFDM system 
International Journal of Wireless \& Mobile Networks (IJWMN) Vol. 5, No. 2, April 2013

After applying the DCT-II on OFDM signal the signal get compressed decreasing the PAPR value. Mucompanding is performed on the I and Q outputs of the IFFT after precoding. As the word length at the IFFT output is decreased, the power consumption and complexity of the DAC/ADC decreases. Also companding at the IFFT output increases the resolution giving a better average signal.

\section{Simulation Results}

To reduce the PAPR, the above discussed has been designed and results are shown in the form of CCDF vs. PAPR plot for different modulations and different values of M. In simulation, an OFDM system is considered under an additive white Gaussian noise (AWGN ) channel, where data is randomly generated with subcarriers $N=2400$, then the signal is modulated by $M-P S K$ and $M-Q A M$ respectively. The PAPR reduction capability is measured by the complementary cumulative distribution $(C C D F=\operatorname{Prob}(P A P R>P A P R O)$, which indicates the probability that PAPR is above a certain threshold. We compared the simulation results of proposed system with DCT-II precoded OFDM signal and precoded $\mu$-Law companded signal. The results were observed for precoded signal and for precoded $\mu$-Law companded signal.

\subsection{M-PSK Modulation}

In this section $\mu$-Law companding technique is applied over DCT-II precoded OFDM signal with phase shift keying modulation. We assume CCDF clip rate of $10^{-0.6}$ using M-ary PSK technique for $\mathrm{N}=2400$ and $\mathrm{M}=16,32,64,128,256,512$ and 1024.

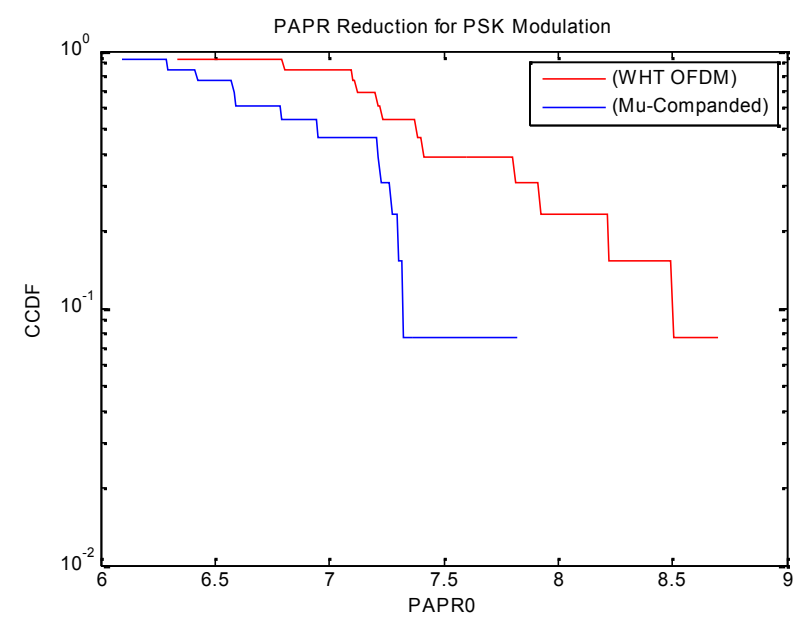

Fig. 3: CCDF of $\mu$-Law companding with proposed DCT-II technique for 16 PSK

For OFDM system CCDF vs. PAPR plots using M=16 over AWGN channel is shown in Fig. 3. The graph gives the clear idea that at CCDF rate of $10^{-0.6}$ the PAPR value decreases by 0.53 $\mathrm{dB}$ using PSK modulation. The proposed precoded $\mu$-Law companded technique provides much reduction in PAPR value as compared to DCT-II precoded signal. 
International Journal of Wireless \& Mobile Networks (IJWMN) Vol. 5, No. 2, April 2013

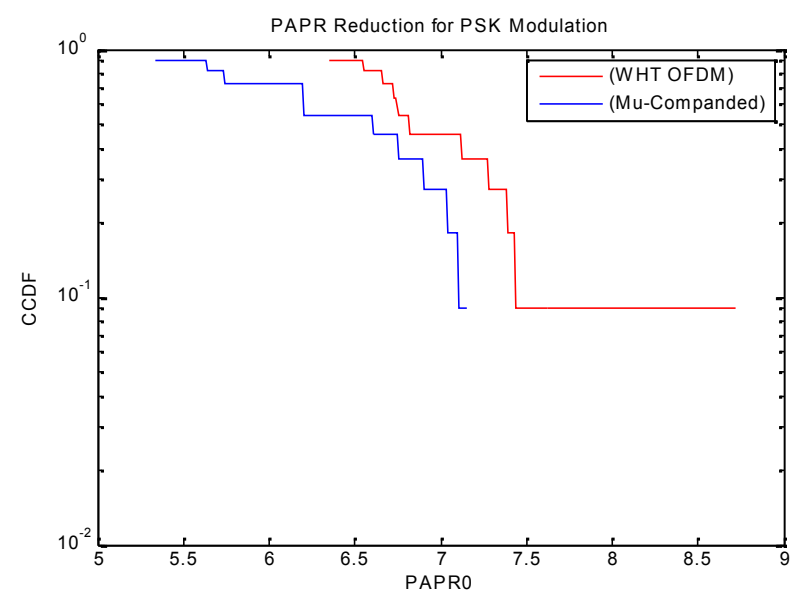

Fig. 4: CCDF of $\mu$-Law companding with proposed DCT-II technique for 32 PSK

For OFDM system CCDF vs. PAPR plots using M=32 over AWGN channel is shown in Fig. 4. The graph gives the clear idea that at CCDF rate of $10^{-0.6}$ the PAPR value decreases by 0.51 $\mathrm{dB}$ using PSK modulation. The proposed precoded $\mu$-Law companded technique provides much reduction in PAPR value as compared to DCT-II precoded signal.

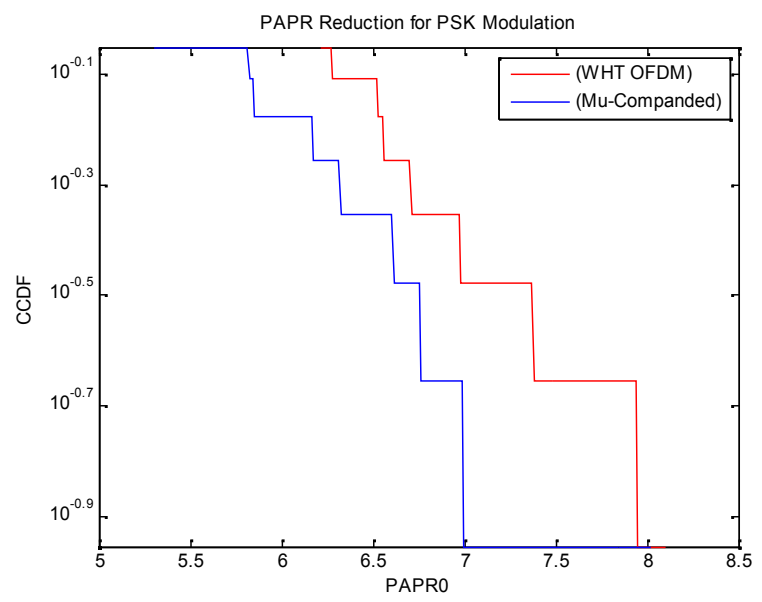

Fig. 5: CCDF of $\mu$-Law companding with proposed DCT-II technique for 64 PSK

For OFDM system CCDF vs. PAPR plots using M=64 over AWGN channel is shown in Fig. 5. The graph gives the clear idea that at CCDF rate of $10^{-0.6}$ the PAPR value decreases by $0.7 \mathrm{~dB}$ using PSK modulation. The proposed precoded $\mu$-Law companded technique provides much reduction in PAPR value as compared to DCT-II precoded signal. 
International Journal of Wireless \& Mobile Networks (IJWMN) Vol. 5, No. 2, April 2013

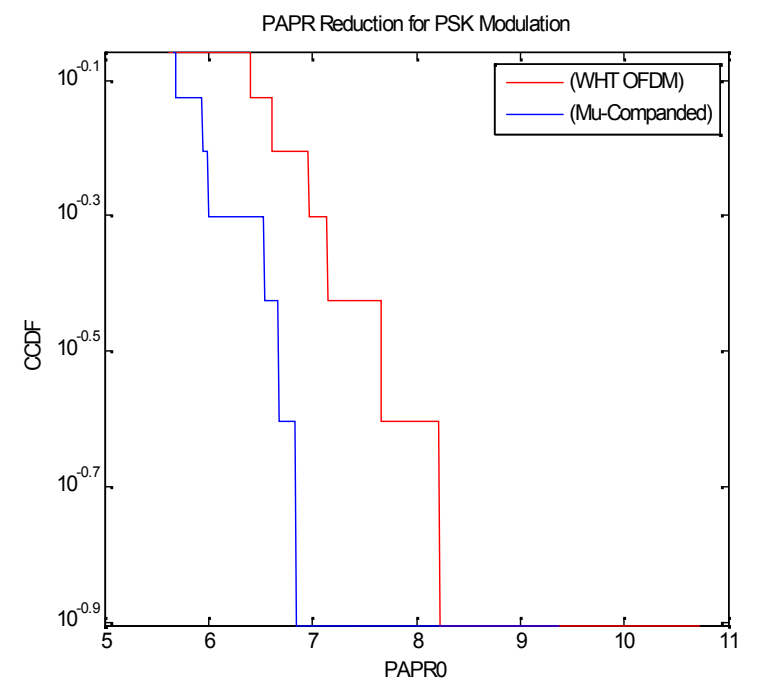

Fig. 6: CCDF of $\mu$-Law companding with proposed DCT-II technique for 128 PSK

For OFDM system CCDF vs. PAPR plots using M=128 over AWGN channel is shown in Fig. 6. The graph gives the clear idea that at CCDF rate of $10^{-0.6}$ the PAPR value decreases by 1.03 $\mathrm{dB}$ using PSK modulation. The proposed precoded $\mu$-Law companded technique provides much reduction in PAPR value as compared to DCT-II precoded signal.

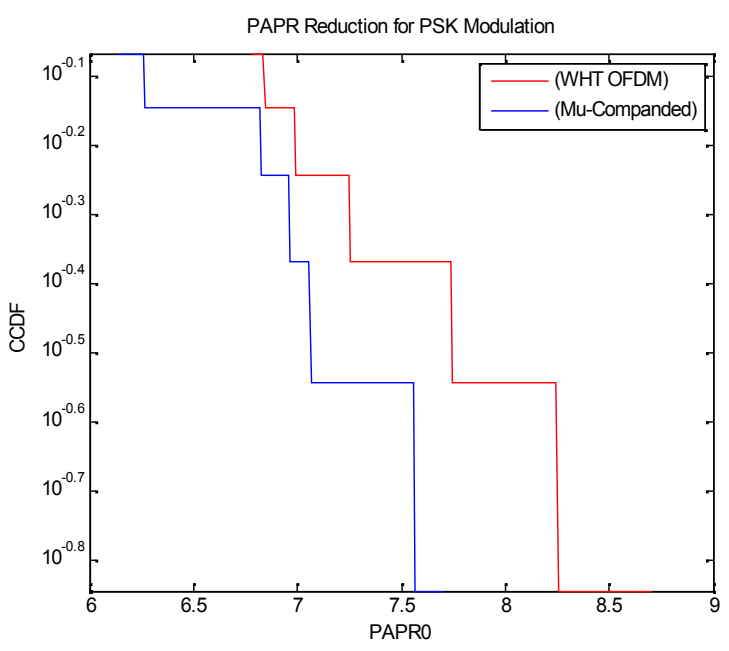

Fig. 7: CCDF of $\mu$-Law companding with proposed DCT-II technique for 256 PSK

For OFDM system CCDF vs. PAPR plots using M=256 over AWGN channel is shown in Fig. 7. The graph gives the clear idea that at CCDF rate of $10^{-0.6}$ the PAPR value decreases by 0.65 $\mathrm{dB}$ using PSK modulation. The proposed precoded $\mu$-Law companded technique provides much reduction in PAPR value as compared to DCT-II precoded signal. 
International Journal of Wireless \& Mobile Networks (IJWMN) Vol. 5, No. 2, April 2013

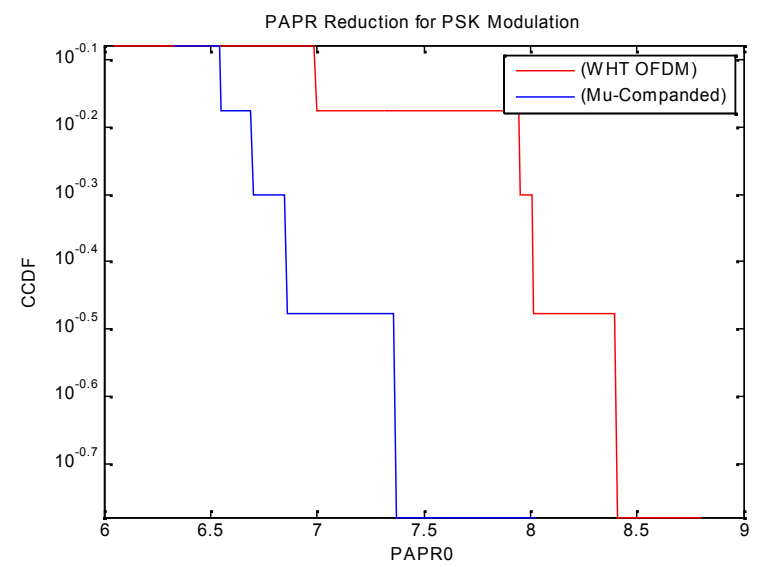

Fig. 8: CCDF of $\mu$-Law companding with proposed DCT-II technique for 512 PSK

For OFDM system CCDF vs. PAPR plots using M=512 over AWGN channel is shown in Fig. 8. The graph gives the clear idea that at CCDF rate of $10^{-0.6}$ the PAPR value decreases by 1.02 $\mathrm{dB}$ using PSK modulation. The proposed precoded $\mu$-Law companded technique provides much reduction in PAPR value as compared to DCT-II precoded signal.

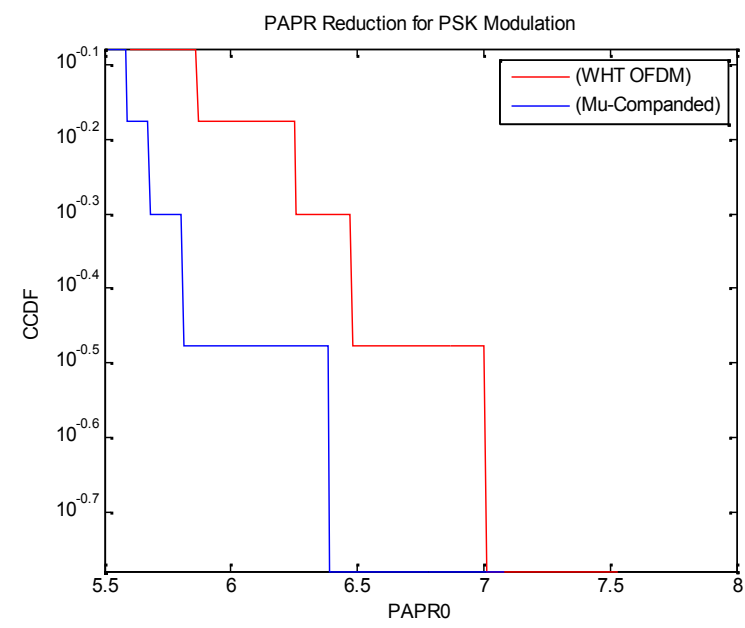

Fig. 9: CCDF of $\mu$-Law companding with proposed DCT-II technique for 1024 PSK

For OFDM system CCDF vs. PAPR plots using M=1024 over AWGN channel is shown in Fig. 9. The graph gives the clear idea that at CCDF rate of $10^{-0.6}$ the PAPR value decreases by 0.62 $\mathrm{dB}$ using PSK modulation. The proposed precoded $\mu$-Law companded technique provides much reduction in PAPR value as compared to DCT-II precoded signal.

\subsection{M-QAM Modulation}

For $\mathrm{M}=16,64,256$ and $1024 \mathrm{M}$-ary quadrature amplitude modulation (QAM) technique is used here at CCDF rate of $10^{-0.6}$. 
International Journal of Wireless \& Mobile Networks (IJWMN) Vol. 5, No. 2, April 2013

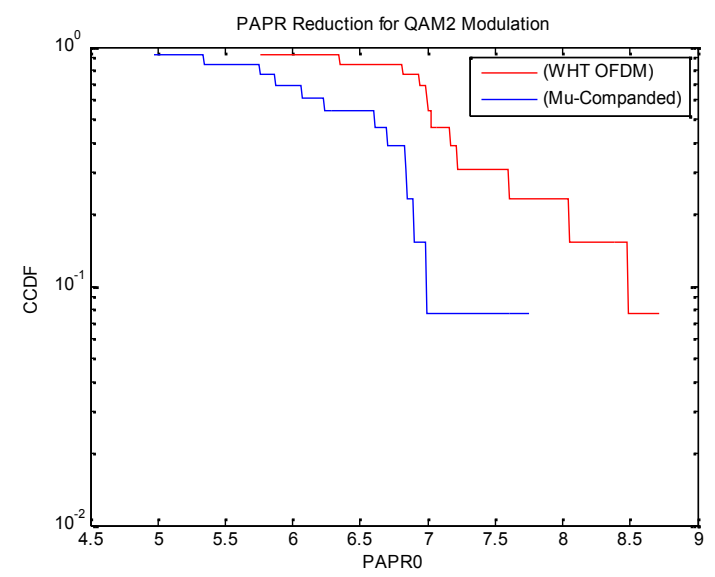

Fig. 10: CCDF of $\mu$-Law companding with proposed DCT-II technique for 16 QAM

For OFDM system CCDF vs. PAPR plots using $\mathrm{M}=16$ over AWGN channel is shown in Fig. 10. The graph gives the clear idea that at CCDF rate of $10^{-0.6}$ the PAPR value decreases by $0.87 \mathrm{~dB}$ using QAM modulation. The proposed precoded $\mu$-Law companded technique provides much reduction in PAPR value as compared to DCT-II precoded signal.

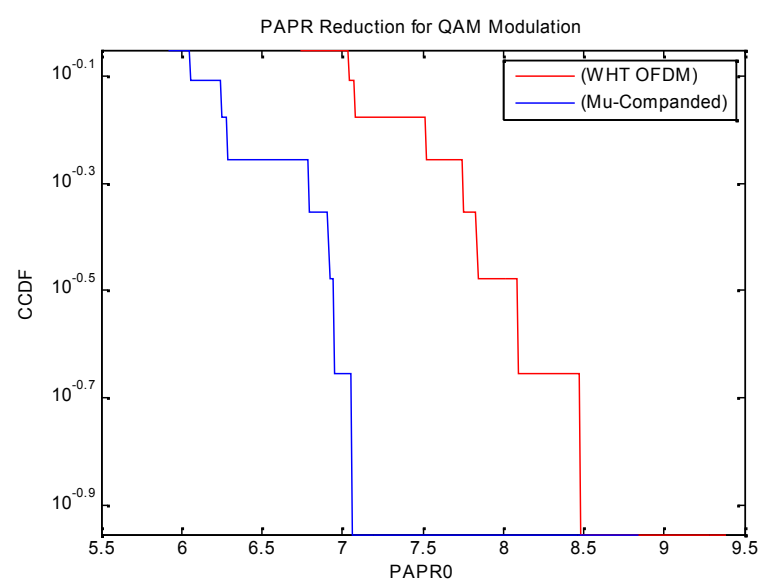

Fig. 11: CCDF of $\mu$-Law companding with proposed DCT-II technique for 64 QAM

For OFDM system CCDF vs. PAPR plots using M=64 over AWGN channel is shown in Fig. 11. The graph gives the clear idea that at CCDF rate of $10^{-0.6}$ the PAPR value decreases by $0.83 \mathrm{~dB}$ using QAM modulation. The proposed $\mu$-Law companded technique provides much reduction in PAPR value as compared to DCT-II precoded signal. 
International Journal of Wireless \& Mobile Networks (IJWMN) Vol. 5, No. 2, April 2013

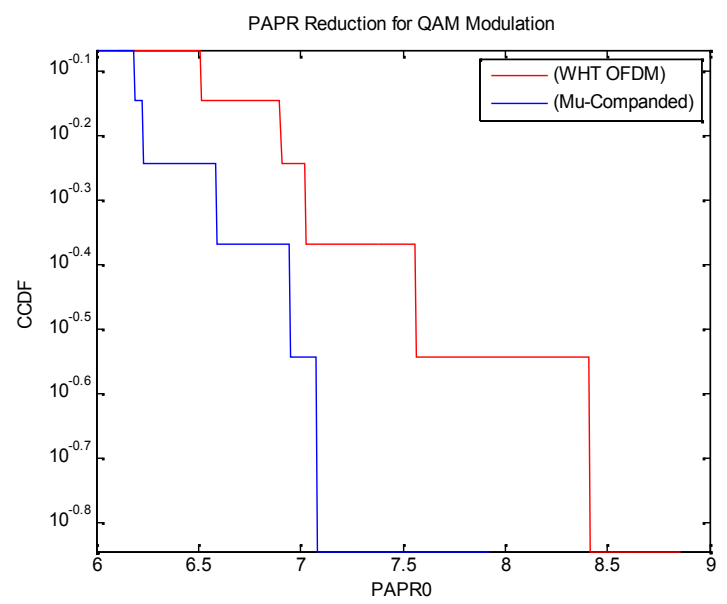

Fig. 12: CCDF of $\mu$-Law companding with proposed DCT-II technique for 256 QAM

For OFDM system CCDF vs. PAPR plots using M=256 over AWGN channel is shown in Fig. 12. The graph gives the clear idea that at CCDF rate of $10^{-0.6}$ the PAPR value decreases by $1.23 \mathrm{~dB}$ using QAM modulation. The proposed precoded $\mu$-Law companded technique provides much reduction in PAPR value as compared to DCT-II precoded signal.

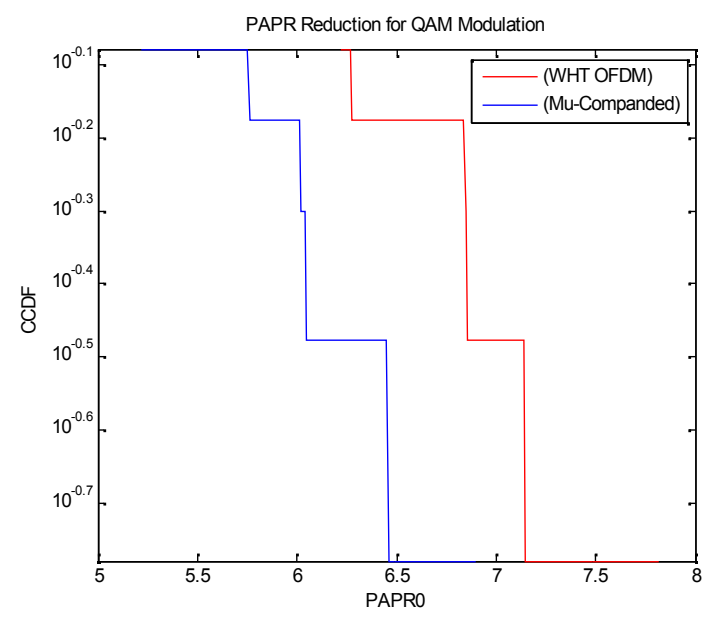

Fig. 13: CCDF of $\mu$-Law companding with proposed DCT-II technique for 1024 QAM

For OFDM system CCDF vs. PAPR plots using M=1024 over AWGN channel is shown in Fig. 13. The graph gives the clear idea that at CCDF rate of $10^{-0.6}$ the PAPR value decreases by 1.3 $\mathrm{dB}$ using QAM modulation. The proposed precoded $\mu$-Law companded technique provides much reduction in PAPR value as compared to DCT-II precoded signal.

\section{Conclusions}

In this paper, a new idea about the performance of the $\mu$-Law companding technique combined with precoded technique is presented for the reduction of PAPR value. The PAPR value can be reduced for different modulation formats and for different values of $\mathrm{M}$ by using proposed 
International Journal of Wireless \& Mobile Networks (IJWMN) Vol. 5, No. 2, April 2013

scheme without increasing the system complexity. The scheme shows superiority in bandwidth compression of signals and powerful performance in the bit-rate reduction. This system produce the lowest PAPR and is efficient, signal independent, distortion less and does not causes any spectrum side-lobes. The simulation results shows, that the proposed precoded combined $\mu$-law companding method displays a better PAPR reduction performance than the simple DCT-II precoded OFDM signal. Thus, it is concluded that the proposed scheme reduces PAPR effectively compared with precoded DCT-II transform.

\section{REFERENCES}

[1] H. Jiang \& P. A. Wilford, "A hierarchical modulation for up grading digital broadcasting systems," IEEE Transaction on Broadcasting, Vol. 51, pp. 222-229, 2005.

[2] P. W. Wolniansky, G. J. Foschini, G. D. Golden \& R. A. Valenzuela, "V-BLAST: an architecture for realizing very high data rates over the rich- scattering wireless channel," In Proceeding of International symposium on Signals, Systems and Electronics, pp. 259-300, 1998.

[3] J. A. C. Bingham, "Multicarrier modulation for data transmission: An idea whose time has come", IEEE Communication Magazine, Vol. 28, Issue: 1, pp. 5-14, 1990.

[4] R. Gross \& D. Veeneman, "Clipping distortion DMT ADSL systems," IEEE Electronics Letters, Vol. 29, pp. 2080- 2081,1993

[5] T. Jiang \& Y. Wu, “An Overview: Peak to Average Power Ratio Reduction Techniques for OFDM Signals,” IEEE Transactions on Broadcasting, Vol. 54, Issue: 2, pp. 257-268, 2008

[6] F. Fiedler, J. Jedwab\& M. G. Parker,“ A framework for the construction of Golay sequences,” IEEE Information Theory, Vol. 54, pp. 3114-3129, 2008.

[7] J. A. Davis \& J. Jedwab, "Peak to mean power control and error correction for OFDM transmission using Golay sequences and Reed-Muller codes," Electronics Letters, Vol. 33, pp. 267-268, 1997.

[8] M. Park, J. Heeyong, J. Cho, D. Hong \& C. Kang, "PAPR Reduction in OFDM Transmission Using Hadamard Transform," IEEE International Conference on Communications, Vol. 1, pp. 430-433, 2000.

[9] R. W. Bauml, R. F. Fischer \& J. B. Huber, "Reducing the Peak to Average Power Ratio of Multicarrier Modulation by Selected Mapping," Electronics Letters, Vol. 32, Issue: 22, pp. 20502057, 1996.

[10] H. Breiling, S.H. H. Muller Weinfurtner\& J.B. Huber, "SLM peak power reduction without explicit side information,” IEEE Communication Letters, Vol. 5, Issue: 6, pp. 239-241, 2001.

[11] S. H. Muller and J. B. Huber, “OFDM Ratio by Optimum Combination of Partial Transmit Sequences,” IEEE Electronic Letters, Vol. 33, Issue: 5, pp. 368-369, 1997.

[12] Agilent Technologies Application Note, "Characterizing Digitally ModulatedSignal with CCDF Curves", 2000.

[13] R.Van Nee and A. de Wild, "Reducing the peaktoaverage power ratio of OFDM,"IEEE Proceeding on Vehicular Technology Conference, pp. 2072-2076, 1998.

[14] H. Ochiai and H. Imai, "On the distribution of thepeak to average power in OFDM signals," IEEE Transactions on Communications, Vol.49, pp.282-289, 2001.

[15] N. T. Ahmed, Natarajan\& K. R. Rao, "Discrete Cosine Transform," IEEE Transactions on Computer, Vol. 23, pp. 90-93,1974. 
International Journal of Wireless \& Mobile Networks (IJWMN) Vol. 5, No. 2, April 2013

[16] L. Kansal, A. Kansal\& K. Singh, “Analysis of Different High Level Modulation Techniques for OFDM System," International Journal of VLSI and Signal Processing Applications, Vol. 1, Issue: $2,2011$.

[17] L. Kansal, A. Kansal\& K. Singh, "Performance Analysis of MIMO-OFDM System Using QOSTBC Code Structure for M-PSK," Signal Processing: An International Journal, Vol. 5, Issue: $2,2011$.

[18] N. Kaur\& L. Kansal, "Reducing the Peak to Average Power Ratio of OFDM Signals through Walsh Hadamard Transform," Global Journal of Researches in Engineering, Vol. 13, Issue: 1, 2013.

[19] N. Kaur\& L. Kansal, "Peak to Average Power Ratio Reduction of OFDM Signal by Combining Clipping withWalsh Hadamard Transform,'International Journal of Wireless\& Mobile Networks, Vol. 5, Issue: 1, 2013.

\section{Authors}

NavneetKaur was born in Kapurthala .She has done her B.Tech degree in Electronics and Communication Engineering from LovelyProfessional University, Phagwara, India. She is currently pursuing her M.Tech degree from Lovely Professional University, Phagwara, India. Her research area includes Digital Communication \& Wireless Communication.

Lavish Kansal was born in Bathinda.He received his B.Tech degree in Electronics and Communication Engineering from PTU, Jalandhar in 2009 and M.E. degree in Electronics and Communication Engineering from Thapar University, Patiala in 2011. He is working as Assistant Professor in the department of Electronics and communication Engineering, Lovely Professional University, Phagwara, India. He has published 15 papers in International journals. His research area includes Digital Signal Processing, Digital Communication \& Wireless Communication.
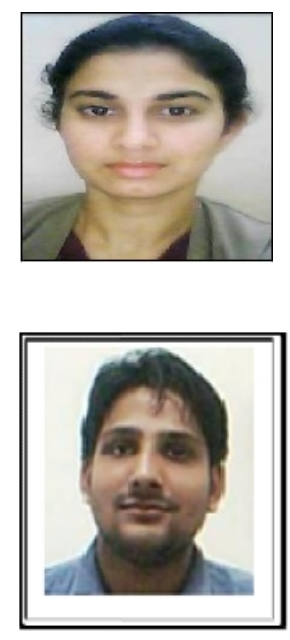